\title{
A Descriptive Study on the Sleeping Habits and Correlation of Sleepiness with Academic Performance in a State University-run Medical School in the Philippines
}

\author{
Manuel Peter Paul C. Jorge II, Ralph Elvi M. Villalobos and Jewel Cordelle C. Nuñal \\ Section of Pulmonary Medicine, Department of Medicine, College of Medicine and Philippine General Hospital, University of the Philippines Manila
}

\begin{abstract}
Background and Significance. Sleep is a vital facet of human existence that is vital to learning and memory; lack of sleep is associated with significant impairment in learning. Medical students are a special population because of the demands of medical school. They are very prone to sleep deprivation and poor quality of sleep, hence academic performance might be affected.
\end{abstract}

Objectives. We determined the different sleeping habits of medical students using a descriptive tool, with variables chosen specifically for this study. The level of sleepiness was then correlated with the academic performance (using the general weighted average) among students in a state university run-medical school in the Philippines.

Methods. The study is a prospective cross-sectional survey among medical students in a state university-run medical school enrolled for the academic year 2016-2017. The questionnaires used were the Epworth Sleepiness Score and specific questions about sleeping habits. The General Weighted Average (GWA) of those who participated were obtained from the student records section of the college. Descriptive statistics were used to describe the results on different sleeping habits, while the chi-squared test was used to determine any significant differences in the GWA versus level of sleepiness across all year levels.

Results. A total of 426 medical students (or $60 \%$ of the total student population of the college) participated. However, of the 426, only 326 had complete GWAs and were therefore included in the final analysis for correlation. The average medical student is "sleep-deprived", sleeping two hours less (six hours) than the recommended daily minimum duration of sleep (eight to 10 hours). For the correlation of sleepiness and academic performance, we found out that there is no significant difference in academic performance among those who are excessively sleepy (ESS greater than 10) versus those who are not, $\mathrm{p}$-value $=0.892$.

Conclusion. Increased level of sleepiness does not correlate with poorer academic performance among these medical students, despite them sleeping less than the general recommendation for adults. The study is limited however by the use of the GWA as the sole tool to measure academic performance, which is affected by many other factors. We recommend the performance of this study in a broader population and use more validated tools to measure sleepiness and academic performance.

Key Words: sleep, sleepiness, academic performance, medical students

\section{BACKGROUND AND SIGNIFICANCE}

Corresponding author: Manuel Peter Paul C. Jorge II, MD Section of Pulmonary Medicine

Department of Medicine

Philippine General Hospital

University of the Philippines Manila

Taft Avenue, Manila 1000, Philippines

Email: mcjorge2@yahoo.com
Sleep is a vital process of human existence. It is defined as unconsciousness from which the person can be aroused by sensory or other stimuli. The principal value of sleep is primarily thought to restore the balances among the neuronal centers, especially in the hippocampus which 
is the primary area for memory and learning. Multiple evidence exists that pathways in the brain that are vital to learning and memory are more active during sleep. In fact, it is often described that the process of learning occurs during waking hours, and sleep functions in the consolidation and storage of these knowledge. ${ }^{1}$

Aside from the learning process, sleep has also a significant effect on mood and other psychosocial aspects of human function, which are also important for the overall well-being of the person. ${ }^{2}$ Sleep deprivation, on the other hand, has been shown to easily disrupt memory consolidation, especially the hippocampal-mediated functions in animal studies. In humans, a reduction in sleeping time is correlated with increased teacher reports of inattention and cognitive problems. ${ }^{2}$ In relation to the academic performance of medical students, a similar study done in Saudi Arabia showed no correlation between increasing sleepiness and academic performance, as measured by the GPA. ${ }^{3}$

The Epworth Sleepiness Scale (ESS) is a short selfadministered questionnaire validated for the diagnosis of excessive sleepiness. ${ }^{3}$ Excessive daytime sleepiness and lack of good quality sleep is associated not only with various cardiometabolic alterations but also with impairment in psychomotor and cognitive function. ${ }^{4}$ The ESS is one of the only various tools to measure sleepiness in the general population. Other tools are available which are more tailored toward symptoms of obstructive sleep apnea, and less specific in diagnosing daytime sleepiness. The relationship of excessive daytime sleepiness as measured by ESS score with other factors such as academic performance is less well-defined.

Tied together with the medical school are lack of sleep and excessive daytime sleepiness because of the heavy academic demands. More so, in the latter years of medical school and towards residency, sleep deprivation is more pronounced because of 24-hour shift hospital duties., ${ }^{5,6}$ This study aims to describe the level of sleepiness and sleeping habits of medical students. Additionally, it also correlated the level of sleepiness with the academic performance of medical students in a state university-run medical school in the Philippines.

\section{REVIEW OF RELATED LITERATURE}

\section{Sleep}

Sleep is a reversible behavioral state of perpetual disengagement from and unresponsiveness to the environment. ${ }^{7}$ Sleep is broadly categorized into two distinct phases, i.e. non-Rapid Eye Movement (NREM) Sleep, which is further subdivided into three phases, and the REM sleep. Normal young adult sleep is usually 7.5 hours and is comprised of $70-80 \%$ NREM sleep. ${ }^{7}$ This normal young adult sleep is disturbed in many medical students and medical residents because of heavy academic demands among the students of the first few years of medical school, and because of 24-hour hospital duties among the more senior students and medical residents.

\section{Sleep Deprivation}

Sleep deprivation is the subjective condition of not having "enough" sleep. It can be sub-classified as being "partial", i.e. the nonsleep stage-specific reduction in total sleep and "total" i.e. having a total loss of sleep. It can be further sub-categorized as either being acute or chronic based on the total number of days of sleep that are affected. ${ }^{7}$ With respect to medical students and residents, the more commonly reported form is chronic, partial loss of sleep (more often in the more junior students), while those in the more physically demanding specialties can suffer total loss of sleep.

The cognitive effects of total and partial sleep deprivation have been more extensively studied with total sleep deprivation. Several studies have already proven the negative effect of total sleep deprivation, showing decreased attention, decreased working memory and decreased vigilance. ${ }^{8}$ In contrast to total sleep deprivation, there are a few published studies regarding the cognitive effects of chronic partial sleep deprivation. These include decreased speed and accuracy, among other detrimental effects. ${ }^{9}$ It is however noteworthy that subjects with chronic partial sleep deprivation have more adverse metabolic effects, including elevated ghrelin and reduced leptin, which may explain the increased BMI among these individuals. ${ }^{10}$

Specifically tackling medical students, a number of published studies have shown that lack of sleep is associated with impaired ability to choose the correct answer in multiple-choice questions and may result in poorer academic performance. ${ }^{3}$ Among resident physicians and consultants who are involved in hospital on-call duties, the effects of lack of sleep include increased error rates in intensive care units, ${ }^{11}$ poorer quality intubations,${ }^{12}$ and lack of empathy towards patients and their relatives. ${ }^{13}$ All of these factors can contribute to poor quality of life among medical students and medical practitioners.

\section{Excessive Daytime Sleepiness and the Epworth Sleepiness Scale}

The ESS is a self-administered questionnaire that was developed in 1991 and is primarily used to diagnose excessive daytime sleepiness from pathologies such as obstructive sleep apnea, narcolepsy, and hypersomnia. ${ }^{3}$ It is a simple questionnaire with eight questions regarding falling asleep in different situations with responses ranging from 0 (low chance of falling asleep) to 3 (high chance of falling asleep). The normal score is less up to 7 , and values greater than 10 indicate excessive daytime sleepiness and warrants a referral to a sleep specialist. Locally, two published studies have shown that among medical residents in Metro Manila, the prevalence of excessive daytime sleepiness was $90.5 \%$, most noted among the first year of residency and in those with prolonged duty hours. The adverse effects of 
this lack of sleep with regard to academic performance and patient care, however, have not been studied. One limitation of the ESS is that it only defines the presence or absence of excessive daytime sleepiness, and is not validated to be correlated with disease entities, cognitive performance, and other psychosocial aspects.

\section{Sleeping habits}

Because sleep is universal, there are also varied experiences associated with it. The term "sleeping habits" are activities that are done before, during, or after the process of sleep which is thought to have contributions to the quality of the sleeping process. There is no available validated tool for sleeping habits as it is a compilation of activities that have not found any firm correlation with the sleep experience. In this study, the "sleeping habits" that we have used in this study are the ones that we deem to be the most commonly encountered by the general population. These specific activities are answerable by yes or no.

To date, no local study has been conducted addressing the prevalence of excessive daytime sleepiness specifically among medical students, and its possible correlation on academic performance.

\section{OBJECTIVES}

\section{General}

To describe the average ESS and most common sleeping habits in medical students of a medical school in the Philippines and correlate the level of sleepiness with the academic performance of medical students in a state university-run medical school in the Philippines.

\section{Specific}

1. To characterize the different sleeping habits of medical students across all year levels.

2. To measure the level of daytime sleepiness among students using the ESS.

3. To correlate the sleeping patterns and ESS scores of medical students with their academic performance using their general weighted average.

\section{MATERIALS AND METHODS}

\section{Study design}

The study is a prospective cross-sectional survey among medical students of all year levels enrolled for the academic year 2016-2017.

\section{Study population}

All medical students who are currently enrolled during the school year of 2016-2017 were invited to participate in the study. The survey was conducted in a state universityrun medical school in the National Capital Region of the Philippines.
This is a study done in the medical school to which the authors were affiliated. Additionally, the authors chose this medical school since the grading system in different medical schools differ and may not be equivalent.

\section{Inclusion criteria}

All enrolled medical students from Learning Unit (LU) 1 (first-year pre-medicine) to LU 6 (hospital clerkship) who consented were included. The authors included all students who gave consent, whether or not they were diagnosed with a sleeping problem or other problems affecting neurocognitive behavior. Those who were taking medications, admit to taking sleeping aids, or stimulants, were also included in the study.

\section{Exclusion criteria}

The only exclusion criteria were students who did not consent, those on leave of absence and those with incomplete grades.

\section{Data collection}

Self-administered questionnaires were distributed to all medical students in the middle of the semester with an informed consent form attached. The questionnaire included the ESS and specific questions on sleeping habits (Appendices A and B).

The general weighted average of the respondents at the end of the academic year (using the standard university grading system) were obtained from the official records of the college was then correlated with the ESS. The grading system of the state university is a numeric system that assigns a grade from 1.0 to 5.0, with increments of 0.25 until 3.0 (the passing mark). The highest grade is 1.0 and the lowest grade is 5.0.

\section{Ethical Issues}

This study was approved prior to the conduct of the study by the Ethics Review Board of the University to which the medical school is attached. The survey was performed in an individual cubicle, with the consent process explained thoroughly to the participant by the authors. Privacy was maintained by not using the full name, but only the student number of the participant, which is not known to the authors. At the collection of the grades, only the student numbers of those who consented were submitted to the college, and the college returned the form with the corresponding GWA in a sealed envelope. The students who were diagnosed to have excessive daytime sleepiness via the ESS were then offered referral to a sleep specialist, for the workup and proper management.

\section{Statistical Analysis}

Descriptive statistics (means) will be used to illustrate the ESS scores and different sleeping habits of these medical students. The cut-off score for the ESS was placed at 10 points, with less than 10 points having no excessive daytime sleepiness. Those whose scores are 10 or greater 
were also grouped and were presumed to have excessive daytime sleepiness. The GWAs were then grouped from 1.00 - 1.75 ("Excellent" -"Very Good"), 1.751 - 2.50 ("Good" - "High Average"), 2.50 - 3.0 ("Average" to "Pass") and $>3.0$ (Failing). The chi-squared test was employed to see the statistical difference between the two groups (i.e. those with or without excessive daytime sleepiness), using alpha significance value of 0.05 .

\section{Limitations}

This study is limited by a variety of factors, especially by the use of the GWA as the measure of "academic performance". The authors recognized the fact that the GWA is affected by a myriad of other factors and is not validated to correlate purely with academic performance. However, the GWA is the only objective measure available, hence it was chosen.

Another limitation is that there are no widely accepted and validated "sleeping habits" that are universal, thus the authors chose the habits that are most commonly encountered in literature. The ESS is also limited by the subjectiveness of the test and is not temporally related to the school activities of the student (i.e. it is taken at one point in time, not necessarily coinciding with the peak or nadir of school activities). Nonetheless, the ESS was chosen because it is the most widely available and validated test to measure excessive daytime sleepiness.

\section{RESULTS}

A total of 426 students participated in the survey (59\% of the total student population of the college) (Table 1).

Table 1. Distribution of study respondents per year level

\begin{tabular}{lr} 
Year Level & Total respondents (\%) \\
LU 1 and 2 (pre-medicine) & $27(6.3)$ \\
LU 3 (first-year medicine proper) & $128(30.1)$ \\
LU 4 (second-year medicine proper) & $121(28.5)$ \\
LU 5 (third-year medicine proper) & $98(23.1)$ \\
LU 6 (hospital clerkship) & $52(12.2)$ \\
\hline Total & $426(100)$ \\
\hline
\end{tabular}

\section{Hours of sleep}

The average medical student sleeps a total of 6.43 hours per night, and the average duration of continuous sleep is 6.40, which is below the current recommended minimum duration of healthy sleep which is eight to 10 hours per night. Across the year levels, self-reported hours of sleep did not differ significantly from LU1 to LU6 ( $p>0.05)$, contrary to what was previously expected to have the hospital clerks sleep less than the more junior medical students (Figure 1).

\section{Specific Sleeping Habits}

Most students are regular coffee takers (57.4\%). The majority of these students also practice good sleep hygiene,

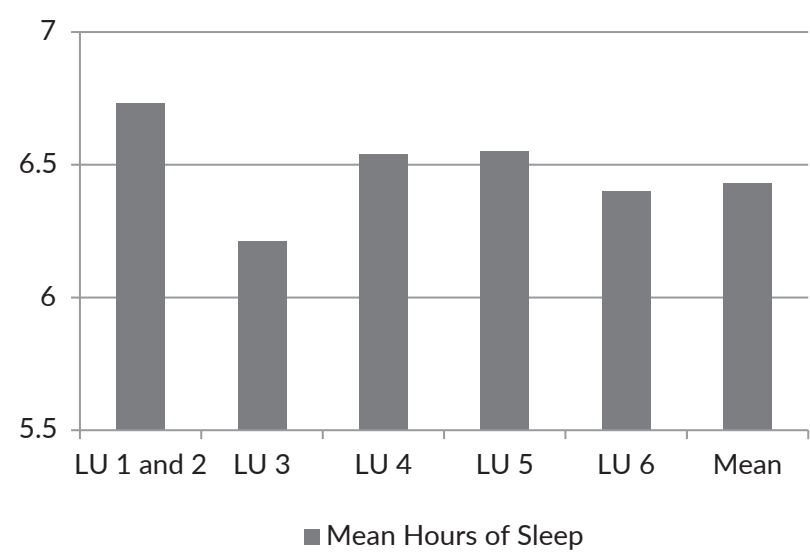

Figure 1. Mean sleep duration did not differ significantly across different year levels.

with students who take a nightly "ritual" and those sleeping with the lights off are outnumbering those who don't. There is however a high number of nighttime awakenings in this population (close to one awakening per night per student). The number of those who need sleeping aids to promote sleep and take nightcaps is only very small $(1.7 \%$ and $0.9 \%$ respectively). Table 2 shows in detail the specific sleeping habits of these medical students.

Table 2. Specific sleeping habits of medical students Specific Sleeping Habit Number (\%)

Students with regular intake of caffeine/nicotine/ 244 (57.4) other stimulants to promote wakefulness

Number of students who sleep with the lights on $\quad 140$ (32.9)

Number of students who eat a heavy meal within $\quad 63(14.8)$ 1 hour from sleeping

Number of students who have a regular sleeping $258(60.7)$ "ritual" before sleep

Number of students who take a "nightcap" 4 (0.9)

Mean number of nighttime awakenings

$354(0.83)$

Intake of sleeping aids to promote sleep

$7(1.7)$

\section{Epworth Sleepiness Score}

The mean ESS is highest in the LU3, or the first year of medicine proper year and declines by the time of the Integrated Clinical Clerkship year (considered the transition year, where students mostly spend their time in the outpatient clinics and have no 24-hour duties). It then increases by the time of clerkship, the first year that hospital duties are being implemented. All mean ESS values are significantly different per year level. These results say that individual medical students sleep significantly longer or shorter compared to each other, and experience a different level of sleepiness from each other, which is a marker of sleep heterogeneity. Nonetheless, at almost all year levels, the mean ESS is greater than 10, which signals that medical students are excessively sleepy during daytime (Figure 2). 


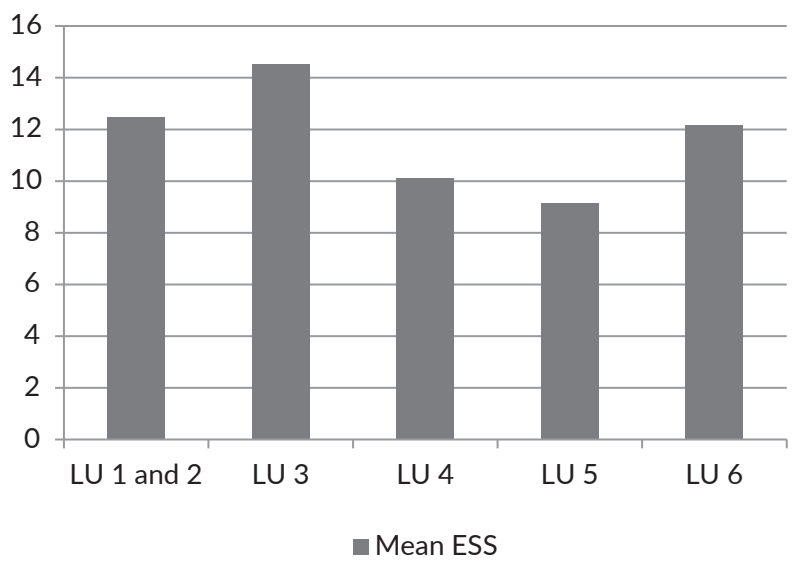

Figure 2. Mean ESS Scores of medical students across all year levels. All p-values are <0.01 for every year level.

\section{Correlation of Epworth Sleepiness Score with General Weighted Average (GWA)}

There are 326 students with complete GWAs and were included in the final analysis. There is no significant difference among grading groups when using the chi-squared statistic $(\mathrm{p}=0.892)$ as shown in Table 3.

Table 3. Comparison of GWA between those with or without excessive daytime sleepiness

\begin{tabular}{lccc} 
GWA & ESS 0-9 & ESS > 10 & $\begin{array}{c}\text { chi-squared } \\
\text { p-value* }\end{array}$ \\
\hline $1.00-1.75$ & 29 & 28 & \\
$1.751-2.50$ & 107 & 129 & 0.892 \\
$2.51-3.00$ & 15 & 16 & \\
$>3.0$ & 1 & 1 & \\
\hline Total & $152(46.6 \%)$ & $174(53.3 \%)$ & \\
\hline
\end{tabular}

\section{DISCUSSION}

The study aimed to describe the different sleeping habits among the medical students of a government medical school in the Philippines. The proportion of medical students with excessive daytime sleepiness was high at $53.3 \%$ and is higher when compared to medical students from other countries like Malaysia (35.5\%), Pakistan (39.5), and Saudi Arabia (37.8\%). ${ }^{3}$

This study confirms that medical students sleep less than the recommended duration for adults. Regarding trends, the study showed that the ESS is highest in the first year of medical school proper. This may be the result of the student's "adjustment period" to the demands of medical school. These first-year medical students also report the shortest duration of sleep, which could also be probably due to this "adjustment period".

Sleeping habits vary considerably. A lot of medical students relied on stimulants to keep them awake. Only a very few required sleeping aids for sleep assistance. These are indicative of good sleep hygiene.
The study showed no difference in the GWA of those who are excessively sleepy versus those who are not. This mirrors the results of a similarly-designed study from Saudi Arabia. ${ }^{3}$ Possible explanations for this could be due to the limitation of the GWA as the sole measure of "academic performance". It is widely accepted that academic performance is a multi-faceted aspect of being a medical student, and includes curricular, extra-curricular and clinical performance, the latter being affected by a variety of factors such as subjectiveness of the grading system and the evaluator. Some reports also state that students can also function well even with few hours of sleep, and can perform tasks satisfactorily.

\section{CONCLUSIONS AND RECOMMENDATIONS}

Upon analysis of GWA and the sleepiness level of these students, we found out that there is no significant difference between the GWA and the level of sleepiness of these medical students. We recommend that a more validated questionnaire (since the ESS is also an imperfect tool) and a larger sample size that will cover all medical students from various medical schools be performed. We also recommend using a more validated tool rather than the GWA to measure academic performance, since this tool is affected by various factors and cannot accurately measure true academic performance.

\section{Statement of Authorship}

All authors participated in protocol development, data collection and analysis. The final version were also reviewed and approved by all the authors.

\section{Author Disclosure}

All authors declared no conflicts of interest.

\section{Funding Source}

The authors self-funded the conduct of the study. No external funding sources were sought.

\section{REFERENCES}

1. Hall J, Guyton A. Guyton and Hall Textbook of Medical Physiology, 13th edition. Saunders; 2015.

2. Dixit A, Thawani R, Goyal A, Vaney N. Psychomotor performance of medical students: effect of 24 hours of sleep deprivation. Indian J Psychol Med. 2012; 34(2):129-32. doi:10.4103/0253-7176.101777.

3. Al-Zahrani J, Aldossari K, Abdulmajeed I, Al-ghamdi S, Al-Shamrani A, Al-Qahtani N. Daytime sleepiness and academic performance among medical students. Health Science J. 2016; 10(3):1-5.

4. Johns MW. A new method for measuring daytime sleepiness: the Epworth Sleepiness Scale. Sleep. 1991; 14(6):540-5.

5. Kasper D, Fauci A, Hauser S, Longo D, Jameson J, Loscalzo J. Harrison's Principles of Internal Medicine, 19th edition. McGrawHill; 2015.

6. Refre G, Jorge M, Capalongan T. Sleepiness and sleeping patterns among residents in training at the University of the PhilippinesPhilippine General Hospital. Phil J Int Med. 2005; 43(5):241-8. 
7. Jorge M, Ledesma-Gamba M, Chavez J, Refre G. Sleepiness and sleeping patterns among internal medicine residents in tertiary care hospitals. Phil J Int Med. 2005; 43(5):235-40.

8. Kryger M, Roth T, Dement W. Principles and Practice of Sleep Medicine, 2nd edition. WB Saunders Company; 1994.

9. Alhola P, Polo-Kantola P. Sleep deprivation: Impact on cognitive performance. Neuropsychiatr Dis Treat. 2007; 3(5):553-67.

10. Dinges DF, Pack F, Williams K, Gillen KA, Powell JW, Ott GE, et al. Cumulative sleepiness, mood disturbance, and psychomotor vigilance performance decrements during a week of sleep restricted to 4-5 hours per night. Sleep. 1997; 20(4):267-77.
11. Taheri S, Lin L, Austin D, Young T, Mignot E. Short sleep duration is associated with reduced leptin, elevated ghrelin, and increased body mass index. PLoS Med. 2004; 1(3): e62.

12. Landrigan CP, Rothschild JM, Cronin JW, Kaushal R, Burdick E, Katz JT, et al. Effect of reducing interns' work hours on serious medical errors in intensive care units. N Engl J Med. 2004; 351(18):1838-48.

13. Smith-Coggins R, Rosekind MR, Hurd S, Buccino KR. Relationship of day versus night sleep to physician performance and mood. Ann Emerg Med. 1994; 24(5):928-34.

\section{APPENDICES}

Appendix A. Questionnaire on Sleeping Habits

\section{PERSONAL INFORMATION}

\begin{tabular}{|l|l|l|l|}
\hline Student Number & Informed Consent & Age: & $\begin{array}{l}\text { Sex } \\
\square \text { Male }\end{array}$ \\
\hline Height: $(\mathrm{m})$ & Weight: $(\mathrm{kg})$ & BMI: $\left(\mathrm{kg} / \mathrm{m}^{2}\right)$ \\
\hline Learning Unit & Date & GWA & (for researchers only): \\
Class Rank
\end{tabular}

Please ANSWER on the blank or PLACE A CHECK on the box before the item that best describes the answer to a specific question:

1. On an average day (i.e. no major examinations, no major evaluation activity the following day), how many hours of sleep do you get? Answer:

2. On an average day (as previous question), what is the usual length (in hours) of the longest continuous, uninterrupted sleep that you get? (continuous sleep: defined as sleep without awakening with return to consciousness)

Answer:

3. Do you regularly ( $>3 x /$ week) take stimulants? (i.e. caffeine, energy drinks, nicotine, etc)
$\square$ YES
$\square$ NO

4. Do you sleep with the lights on?
$\square$ YES
NO

5. Do you eat a heavy meal within 1 hour from sleeping?
$\square$ YES
$\square$ NO

6. Do you practice a regular "ritual" before sleeping? (ritual: anything from taking a bath, washing face, praying, etc that you regularly do before sleeping)
$\square$ YES
NO

7. Do you take a "nightcap" before sleeping? (nightcap: most commonly alcoholic beverages taken with the intention to promote sleeping)
$\square$ YES
$\square \mathrm{NO}$

8. On a regular day, how many awakenings do you recall to have in your sleep?

Answer:

9. Do you take sleeping aids to help promote sleep?
$\square$ YES
$\square \mathrm{NO}$

Thank you very much for participating in our research!

Accomplished by (Signature over printed name):

Date: 
Appendix B. The Epworth Sleepiness Scale (ESS)

\section{INTRODUCTION}

The Epworth Sleepiness Scale is widely used in the field of sleep medicine as a subjective measure of a patient's sleepiness. The test is a list of eight situations in which you rate your tendency to become sleepy on a scale of $\mathbf{0}$ (no chance of sleeping) to 3 (high chance of sleeping). Your total score is based on a scale of 0 to 24. The scale estimates whether you are experiencing excessive sleepiness that possibly requires medical attention.

\section{HOW SLEEPY ARE YOU?}

How likely are you to doze off or fall asleep in the following situations?

You should rate your chances of dozing off, not just feeling tired. Even if you have not done some of these things recently try to determine how they would have affected you.

For each situation, decide whether or not you would have:

\section{$0=$ NO chance of sleeping \\ 1 = SLIGHT chance of sleeping \\ 2 = MODERATE chance of sleeping \\ $3=$ HIGH chance of sleeping}

Write down the number corresponding to your choice in the right hand column. Total your score below.

\begin{tabular}{|l|l|}
\multicolumn{1}{|c|}{ SITUATION } & \\
\hline Sitting down and reading & \\
\hline Watching television & \\
\hline Sitting inactive in a public place (e.g. in a theater, in a meeting) & \\
\hline As a passenger in a car for an hour without a break & \\
\hline Lying down to rest in the afternoon when circumstances permit & \\
\hline Sitting down and talking to someone & \\
\hline Sitting down quietly after lunch without alcohol & \\
\hline In a car, stopped for a few minutes in traffic & TOTAL \\
\hline
\end{tabular}

Accomplished by (printed name and signature):

Date:

Thank you very much for participating! 